Article

\title{
Boundary Value Problems for Hilfer Fractional Differential Inclusions with Nonlocal Integral Boundary Conditions
}

\author{
Athasit Wongcharoen ${ }^{1,+}$, Sotiris K. Ntouyas ${ }^{2,3,+}$ (D) and Jessada Tariboon ${ }^{4, *,+(D)}$ \\ 1 Department of Mechanical Engineering Technology, College of Industrial Engineering Technology, \\ King Mongkut's University of Technology North Bangkok, Bangkok 10800, Thailand; \\ athasit.w@cit.kmutnb.ac.th \\ 2 Department of Mathematics, University of Ioannina, 45110 Ioannina, Greece; sntouyas@uoi.gr \\ 3 Nonlinear Analysis and Applied Mathematics (NAAM)-Research Group, Department of Mathematices, \\ Faculty of Science, King Abdulaziz University, P.O. Box 80203, Jeddah 21589, Saudi Arabia \\ 4 Intelligent and Nonlinear Dynamic Innovations Research Center, Department of Mathematics, \\ Faculty of Applied Science, King Mongkut's University of Technology North Bangkok, \\ Bangkok 10800, Thailand \\ * Correspondence: jessada.t@sci.kmutnb.ac.th \\ + These authors contributed equally to this work.
}

Received: 2 October 2020; Accepted: 28 October 2020; Published: 31 October 2020 updates

\begin{abstract}
In this paper, we study boundary value problems for differential inclusions, involving Hilfer fractional derivatives and nonlocal integral boundary conditions. New existence results are obtained by using standard fixed point theorems for multivalued analysis. Examples illustrating our results are also presented.
\end{abstract}

Keywords: Hilfer fractional derivative; Riemann-Liouville fractional derivative; Caputo fractional derivative; boundary value problems; existence; fixed point theory

\section{Introduction}

The theory of fractional differential equations received in recent years considerable interest both in pure mathematics and applications, see [1-9]. In the literature, there exist several different definitions of fractional integrals and derivatives, for example, the most popular of them are fractional derivatives in the sense of Riemann-Liouville and Caputo. Other known definitions are the Hadamard fractional derivative, the Erdeyl-Kober fractional derivative, and so on. We refer the interested in fractional calculus reader to the classical reference texts such as [1-7]. A generalization of derivatives of both Riemann-Liouville and Caputo was given by R. Hilfer in [10] when he studied fractional time evolution in physical phenomena. He named it as generalized fractional derivative of order $\alpha \in(0,1)$ and a type $\beta \in[0,1]$ which can be reduced to the Riemann-Liouville and Caputo fractional derivatives when $\beta=0$ and $\beta=1$, respectively. Such a derivative interpolates between the Riemann-Liouville and Caputo derivative. Some properties and applications of the Hilfer derivative are given in $[11,12]$ and references cited therein.

Initial value problems involving Hilfer fractional derivatives were studied by several authors, see for example [13-15] and references therein. Recently, in [16], the authors initiated the study of boundary value problems for Hilfer fractional differential equations with nonlocal integral boundary conditions of the form

$$
{ }^{H} D^{\alpha, \beta} x(t)=f(t, x(t)), \quad t \in J:=[a, b], \quad 1<\alpha<2,0 \leq \beta \leq 1,
$$




$$
x(a)=0, \quad x(b)=\sum_{i=1}^{m} \delta_{i} I^{\varphi_{i}} x\left(\xi_{i}\right), \quad \varphi_{i}>0, \delta_{i} \in \mathbb{R}, \xi_{i} \in[a, b],
$$

where ${ }^{H} D^{\alpha, \beta}$ is the Hilfer fractional derivative of order $\alpha, 1<\alpha<2$ and parameter $\beta, 0 \leq \beta \leq 1, I^{\varphi_{i}}$ is the Riemann-Liouville fractional integral of order $\varphi_{i}>0, \xi_{i} \in J, a \geq 0, \delta_{i} \in \mathbb{R}$, and $f: J \times \mathbb{R} \rightarrow \mathbb{R}$ is a continuous function. Several existence and uniqueness results were proved by using a variety of fixed point theorems, such as Banach's fixed point theorem, Hölder's inequality, and Boyd and Wong fixed point theorem for nonlinear contractions to obtain the uniqueness results, while nonlinear alternative of Leray-Schauder type and Krasnoselskii's fixed point theorem were applied to obtain the existence results. For some other recent results, see [17,18].

The objective of the present work is to study the multivalued case of the problem (1) and (2); that is, the boundary value problem of Hilfer-type fractional differential inclusions with nonlocal integral boundary conditions

$$
\begin{aligned}
& { }^{H} D^{\alpha, \beta} x(t) \in F(t, x(t)), \quad t \in J, \quad 1<\alpha<2, \quad 0 \leq \beta \leq 1, \\
& x(a)=0, \quad x(b)=\sum_{i=1}^{m} \delta_{i} I^{\varphi_{i}} x\left(\xi_{i}\right), \quad \varphi_{i}>0, \delta_{i} \in \mathbb{R}, \xi_{i} \in[a, b],
\end{aligned}
$$

where $F: J \times \mathbb{R} \rightarrow \mathcal{P}(\mathbb{R})$ is a multivalued map, $\mathcal{P}(\mathbb{R})$ is the family of all nonempty subsets of $\mathbb{R}$, and the other parameters are as in problem (1) and (2).

We obtain existence results for the problem (3) and (4) by applying the nonlinear alternative of Leray-Schauder type, and a fixed point theorem due to Nadler. Examples are also constructed for the illustration of main results.

The rest of the paper is arranged as follows. In Section 2, we recall some preliminary concepts of fractional calculus and multivalued analysis. Section 3 is devoted to the main results, while examples illustrating the obtained results are presented in Section 4.

\section{Preliminaries}

In this section, we introduce some notation and definitions of fractional calculus and present preliminary results needed in our proofs later [2,5].

Definition 1. The Riemann-Liouville fractional integral of order $\alpha>0$ of a continuous function is defined by

$$
I^{\alpha} u(t)=\frac{1}{\Gamma(\alpha)} \int_{a}^{t}(t-s)^{\alpha-1} u(s) d s, \quad n-1<\alpha<n,
$$

where $n=[\alpha]+1,[\alpha]$ denotes the integer part of real number $\alpha$, provided the right-hand side is point-wise defined on $(a, \infty)$, where $\Gamma(\cdot)$ is the Euler gamma function, which is defined by $\Gamma(r)=\int_{0}^{\infty} t^{r-1} e^{-t} d t$.

Definition 2. The Riemann-Liouville fractional derivative of order $\alpha>0$ of a continuous function is defined by

$$
{ }^{R L} D^{\alpha} u(t):=D^{n} I^{n-\alpha} u(t)=\frac{1}{\Gamma(n-\alpha)}\left(\frac{d}{d t}\right)^{n} \int_{a}^{t}(t-s)^{n-\alpha-1} u(s) d s, n-1<\alpha<n,
$$

where $n=[\alpha]+1$, provided the right-hand side is point-wise defined on $(a, \infty)$.

Definition 3. The Caputo fractional derivative of order $\alpha>0$ of a continuous function is defined by

$$
{ }^{C} D^{\alpha} u(t):=I^{n-\alpha} D^{n} u(t)=\frac{1}{\Gamma(n-\alpha)} \int_{a}^{t}(t-s)^{n-\alpha-1}\left(\frac{d}{d s}\right)^{n} u(s) d s, n-1<\alpha<n,
$$

provided the right-hand side is point-wise defined on $(a, \infty)$. 
In [10] (see also [12]), another new definition of the fractional derivative was suggested. The generalized Riemann-Liouville fractional derivative defined as

Definition 4. The generalized Riemann-Liouville fractional derivative or Hilfer fractional derivative of order $\alpha$ and parameter $\beta$ of a function is defined by

$$
{ }^{H} D^{\alpha, \beta} u(t)=I^{\beta(n-\alpha)} D^{n} I^{(1-\beta)(n-\alpha)} u(t),
$$

where $n-1<\alpha<n, 0 \leq \beta \leq 1, t>a, D=\frac{d}{d t}$.

Remark 1. When $\beta=0$, the Hilfer fractional derivative corresponds to the Riemann-Liouville fractional derivative

$$
{ }^{H} D^{\alpha, 0} u(t)=D^{n} I^{n-\alpha} u(t),
$$

while when $\beta=1$ the Hilfer fractional derivative corresponds to the Caputo fractional derivative

$$
{ }^{H} D^{\alpha, 1} u(t)=I^{n-\alpha} D^{n} u(t)
$$

In the following lemma we present the compositional property of Riemann-Liouville fractional integral operator with the Hilfer fractional derivative operator.

Lemma 1 ([12]). Let $f \in L(a, b), n-1<\alpha \leq n, n \in \mathbb{N}, 0 \leq \beta \leq 1, I^{(n-\alpha)(1-\beta)} f \in A C^{k}[a, b]$. Then

$$
\left(I^{\alpha H} D^{\alpha, \beta} f\right)(t)=f(t)-\sum_{k=0}^{n-1} \frac{(t-a)^{k-(n-\alpha)(1-\beta)}}{\Gamma(k-(n-\alpha)(1-\beta)+1)} \lim _{t \rightarrow a^{+}}\left(I^{(1-\beta)(n-\alpha)} f\right)(t) .
$$

The following lemma deals with a linear variant of the boundary value problem (3) and (4) and is proved in [16].

Lemma 2. Let $\varphi_{i}>0, \xi_{i} \in[a, b], a \geq 0, \delta_{i} \in \mathbb{R}, 1<\alpha<2, \gamma=\alpha+2 \beta-\alpha \beta, \Lambda \neq 0$, and $h \in C([a, b], \mathbb{R})$. Then the function $x$ is a solution of the boundary value

$$
\begin{aligned}
& { }^{H} D^{\alpha, \beta} x(t)=h(t), \quad t \in[a, b], \quad 1<\alpha<2, \quad 0 \leq \beta \leq 1, \\
& x(a)=0, \quad x(b)=\sum_{i=1}^{m} \delta_{i} I^{\varphi_{i}} x\left(\xi_{i}\right), \quad \varphi_{i}>0, \quad \delta_{i} \in \mathbb{R}, \quad \xi_{i} \in[a, b],
\end{aligned}
$$

if and only if

$$
\left.x(t)=\frac{(t-a)^{\gamma-1}}{\Lambda \Gamma(\gamma)}\left(I^{\alpha} h(b)\right)-\sum_{i=1}^{m} \delta_{i} I^{\alpha+\varphi_{i}} h\left(\xi_{i}\right)\right)+I^{\alpha} h(t),
$$

where

$$
\Lambda=\sum_{i=1}^{m} \frac{\delta_{i}\left(\xi_{i}-a\right)^{\gamma+\varphi_{i}-1}}{\Gamma\left(\gamma+\varphi_{i}\right)}-\frac{(b-a)^{\gamma-1}}{\Gamma(\gamma)}
$$

\section{Existence Results}

By $C(J, \mathbb{R})$, we denote the Banach space of all continuous functions from $J$ into $\mathbb{R}$ with the norm

$$
\|x\|:=\sup \{|x(t)|: t \in J\} .
$$

Additionally by $L^{1}(J, \mathbb{R})$, we denote the space of functions $x: J \rightarrow \mathbb{R}$ such that $\|x\|_{L^{1}}=\int_{1}^{b}|x(t)| d t$. 
For a normed space $(X,\|\cdot\|)$, we define: $\mathcal{P}(X)=\{Y \subset X: Y \neq \varnothing\} ; \mathcal{P}_{c p}(X)=\{Y \in$ $\mathcal{P}(X): Y$ is compact $\} ; \mathcal{P}_{c, c p}(X)=\{Y \in \mathcal{P}(X): Y$ is convex and compact $\} ; \mathcal{P}_{b, c l}(X)=\{Y \in \mathcal{P}(X):$ $Y$ is bounded and closed $\}$ and $\mathcal{P}_{c l}(X)=\{Y \in \mathcal{P}(X): Y$ is closed $\}$.

For the basic concepts of multivalued analysis we refer to [19,20]. See also [21].

For each $y \in C(J, \mathbb{R})$, define the set of selections of $F$ by

$$
S_{F, x}:=\left\{v \in L^{1}(J, \mathbb{R}): v(t) \in F(t, y(t)) \text { on } J\right\}
$$

A multivalued map $G: X \rightarrow \mathcal{P}(X)$ has a fixed point if there is $x \in X$ such that $x \in G(x)$. The fixed point set of the multivalued operator $G$ will be denoted by Fix $G$.

Definition 5. A function $x \in C(J, \mathbb{R})$ is said to be a solution of the problem (3) and (4) if there exists a function $v \in L^{1}(J, \mathbb{R})$ with $v(t) \in F(t, x)$ for a.e. $t \in J$ such that $x$ satisfies the differential equation ${ }^{H} D^{\alpha, \beta} x(t)=v(t)$ on $J$ and the boundary conditions $x(a)=0, x(b)=\sum_{i=1}^{m} \delta_{i} I^{\varphi_{i}} x\left(\xi_{i}\right)$.

Definition 6. A multivalued map $F: J \times \mathbb{R} \rightarrow \mathcal{P}(\mathbb{R})$ is said to be Carathéodory if

(i) $\quad t \longmapsto F(t, x)$ is measurable for each $x \in \mathbb{R}$;

(ii) $x \longmapsto F(t, x)$ is upper semicontinuous for almost all $t \in J$. Further a Carathéodory function $F$ is called $L^{1}$-Carathéodory if

(iii) for each $\rho>0$, there exists $\varphi_{\rho} \in L^{1}\left(J, \mathbb{R}^{+}\right)$such that

$$
\|F(t, x)\|=\sup \{|v|: v \in F(t, x)\} \leq \varphi_{\rho}(t)
$$

for all $x \in \mathbb{R}$ with $\|x\| \leq \rho$ and for a.e. $t \in J$.

Our first existence result, dealing with the case when $F$ has convex values, is based on nonlinear alternative of Leray-Schauder type [22] with the assumption that $F$ is Carathéodory. For quick reference, we state the well-known nonlinear alternative of Leray-Schauder for multivalued maps.

Lemma 3. (Nonlinear alternative for Kakutani maps [22]). Let E be a Banach space, C a closed convex subset of $E, U$ an open subset of $C$ and $0 \in U$. Suppose that $F: \bar{U} \rightarrow \mathcal{P}_{c p, c}(C)$ is an upper semicontinuous compact map. Then either

(i) F has a fixed point in $\bar{U}$, or

(ii) there is $a u \in \partial U$ and $\lambda \in(0,1)$ with $u \in \lambda F(u)$.

Theorem 1. Assume that:

$\left(H_{1}\right) F: J \times \mathbb{R} \rightarrow \mathcal{P}_{c p, c}(\mathbb{R})$ is $L^{1}$-Carathéodory;

$\left(H_{2}\right)$ there exists a continuous nondecreasing function $\psi:[0, \infty) \rightarrow(0, \infty)$ and a function $p \in C\left(J, \mathbb{R}^{+}\right)$ such that

$$
\|F(t, x)\|_{\mathcal{P}}:=\sup \{|x|: x \in F(t, x)\} \leq p(t) \psi(\|x\|) \text { for each }(t, x) \in J \times \mathbb{R}
$$

$\left(H_{3}\right)$ there exists a constant $M>0$ such that

$$
\frac{M}{\|p\| \psi(M)\left\{\frac{(b-a)^{\alpha}}{\Gamma(\alpha+1)}+\frac{(b-a)^{\gamma-1}}{|\Lambda| \Gamma(\gamma)}\left(\frac{(b-a)^{\alpha}}{\Gamma(\alpha+1)}+\sum_{i=1}^{m}\left|\delta_{i}\right| \frac{\left(\xi_{i}-a\right)^{\alpha+\varphi_{i}}}{\Gamma\left(\alpha+\varphi_{i}+1\right)}\right)\right\}}>1
$$

Then the boundary value problem (3) and (4) has at least one solution on $[a, b]$. 
Proof. To transform the problem (3) and (4) into a fixed point problem, we define an operator $\mathcal{F}$ : $C([a, b], \mathbb{R}) \longrightarrow \mathcal{P}(C([a, b], \mathbb{R}))$ by

$$
\mathcal{F}(x)=\left\{h \in C([a, b], \mathbb{R}): h(t)=\frac{(t-a)^{\gamma-1}}{\Lambda \Gamma(\gamma)}\left(I^{\alpha} v(b)-\sum_{i=1}^{m} \delta_{i} I^{\alpha+\varphi_{i}} h\left(\xi_{i}\right)\right)+I^{\alpha} v(t)\right\}
$$

for $t \in[a, b]$ and $v \in S_{F, x}$. It is obvious that the fixed points of $\mathcal{F}$ are solutions of the boundary value problem (3) and (4).

We will show that the operator $\mathcal{F}$ satisfies the assumptions of Leray-Schauder nonlinear alternative [22] in a series of steps.

Step 1. $\mathcal{F}(x)$ is convex for each $x \in C([a, b], \mathbb{R})$.

This step is obvious since $S_{F, x}$ is convex ( $F$ has convex values), and therefore we omit the proof.

Step 2. $\mathcal{F}$ maps bounded sets (balls) into bounded sets in $C([a, b], \mathbb{R})$.

Let $B_{r}=\{x \in C([a, b], \mathbb{R}):\|x\| \leq r\}$ be a bounded set in $C([a, b], \mathbb{R})$. Then, for each $h \in \mathcal{B}(x), x \in$ $B_{r}$, there exists $v \in S_{F, x}$ such that

$$
h(t)=I^{\alpha} v(s)(t)+\frac{(t-a)^{\gamma-1}}{\Lambda \Gamma(\gamma)}\left(I^{\alpha} v(s)(b)-\sum_{i=1}^{m} \delta_{i} I^{\alpha+\varphi_{i}} v(s)\left(\xi_{i}\right)\right) .
$$

Then, for $t \in J$, we have

$$
\begin{aligned}
|h(t)| & \leq I^{\alpha}|v(s)|(b)+\frac{(b-a)^{\gamma-1}}{|\Lambda| \Gamma(\gamma)}\left(I^{\alpha}|v(s)|(b)+\sum_{i=1}^{m} \delta_{i} I^{\alpha+\varphi_{i}}|v(s)|\left(\xi_{i}\right)\right) \\
& \leq\|p\| \psi(\|x\|)\left\{\frac{(b-a)^{\alpha}}{\Gamma(\alpha+1)}+\frac{(b-a)^{\gamma-1}}{|\Lambda| \Gamma(\gamma)}\left(\frac{(b-a)^{\alpha}}{\Gamma(\alpha+1)}+\sum_{i=1}^{m}\left|\delta_{i}\right| \frac{\left(\xi_{i}-a\right)^{\alpha+\varphi_{i}}}{\Gamma\left(\alpha+\varphi_{i}+1\right)}\right)\right\} .
\end{aligned}
$$

Thus,

$$
\|h\| \leq\|p\| \psi(r)\left\{\frac{(b-a)^{\alpha}}{\Gamma(\alpha+1)}+\frac{(b-a)^{\gamma-1}}{|\Lambda| \Gamma(\gamma)}\left(\frac{(b-a)^{\alpha}}{\Gamma(\alpha+1)}+\sum_{i=1}^{m}\left|\delta_{i}\right| \frac{\left(\xi_{i}-a\right)^{\alpha+\varphi_{i}}}{\Gamma\left(\alpha+\varphi_{i}+1\right)}\right)\right\} .
$$

Step 3. $\mathcal{F}$ maps bounded sets into equicontinuous sets of $C([a, b], \mathbb{R})$.

Let $\tau_{1}, \tau_{2} \in[a, b]$ with $\tau_{1}<\tau_{2}$ and $x \in B_{r}$. Then, for each $h \in \mathcal{B}(x)$, we obtain

$$
\begin{aligned}
& \left|h\left(t_{2}\right)-h\left(t_{1}\right)\right| \\
\leq & \frac{\left(\tau_{2}-a\right)^{\gamma-1}-\left(\tau_{1}-a\right)^{\gamma-1}}{|\Lambda| \Gamma(\gamma)} I^{\alpha}|v(s)|(b) \\
& +\frac{\left(\tau_{2}-a\right)^{\gamma-1}-\left(\tau_{1}-a\right)^{\gamma-1}}{|\Lambda| \Gamma(\gamma)} \sum_{i=1}^{m}\left|\delta_{i}\right| I^{\alpha+\varphi_{i}}|v(s)|\left(\xi_{i}\right) \\
& +\frac{1}{\Gamma(\alpha)}\left|\int_{a}^{\tau_{1}}\left[\left(\tau_{2}-s\right)^{\alpha-1}-\left(\tau_{1}-s\right)^{\alpha-1}\right] v(s) d s+\int_{\tau_{1}}^{\tau_{2}}\left(\tau_{2}-s\right)^{\alpha-1} v(s) d s\right| \\
\leq & \frac{\left(\tau_{2}-a\right)^{\gamma-1}-\left(\tau_{1}-a\right)^{\gamma-1}}{|\Lambda| \Gamma(\gamma)} \psi(r) I^{\alpha} p(s)(b) \\
& +\frac{\left(\tau_{2}-a\right)^{\gamma-1}-\left(\tau_{1}-a\right)^{\gamma-1}}{|\Lambda| \Gamma(\gamma)} \psi(r) \sum_{i=1}^{m}\left|\delta_{i}\right| I^{\alpha+\varphi_{i}} p(s)\left(\xi_{i}\right)
\end{aligned}
$$




$$
\begin{aligned}
& +\frac{\psi(r)}{\Gamma(\alpha)}\left|\int_{a}^{\tau_{1}}\left[\left(\tau_{2}-s\right)^{\alpha-1}-\left(\tau_{1}-s\right)^{\alpha-1}\right] p(s) d s+\int_{\tau_{1}}^{\tau_{2}}\left(\tau_{2}-s\right)^{\alpha-1} p(s) d s\right| \\
\leq & \frac{\left(\tau_{2}-a\right)^{\gamma-1}-\left(\tau_{1}-a\right)^{\gamma-1}}{|\Lambda| \Gamma(\gamma)}\|p\| \psi(r)\left\{\frac{(b-a)^{\alpha}}{\Gamma(\alpha+1)}+\sum_{i=1}^{m} \frac{\left|\delta_{i}\right|\left(\xi_{i}-a\right)^{\alpha+\varphi_{i}}}{\Gamma\left(\alpha+\varphi_{i}+1\right)}\right\} \\
& +\frac{\|p\| \psi(r)}{\Gamma(\alpha+1)}\left[2\left(\tau_{2}-\tau_{1}\right)^{\alpha}+\left|\left(\tau_{2}-a\right)^{\alpha}-\left(\tau_{1}-a\right)^{\alpha}\right|\right] .
\end{aligned}
$$

Obviously the right hand side of the above inequality tends to zero independently of $x \in B_{r}$ as $\tau_{2}-$ $\tau_{1} \rightarrow 0$. Therefore, it follows by the Arzelá-Ascoli theorem that $\mathcal{F}: C([a, b], \mathbb{R}) \rightarrow \mathcal{P}(C([a, b], \mathbb{R}))$ is completely continuous.

Next we show that $\mathcal{F}$ is an upper semi-continuous multivalued mapping. It is known by [19] (Proposition 1.2) that $\mathcal{F}$ will be upper semicontinuous if we establish that it has a closed graph. Thus, in the next step we show that $\mathcal{F}$ has a closed graph.

Step 4. F has a closed graph.

Let $x_{n} \rightarrow x_{*}, h_{n} \in \mathcal{F}\left(x_{n}\right)$ and $h_{n} \rightarrow h_{*}$. Then we need to show that $h_{*} \in \mathcal{F}\left(x_{*}\right)$. Associated with $h_{n} \in \mathcal{F}\left(x_{n}\right)$, there exists $v_{n} \in S_{F, x_{n}}$ such that for each $t \in[a, b]$,

$$
h_{n}(t)=I^{\alpha} v_{n}(s)(t)+\frac{(t-a)^{\gamma-1}}{\Lambda \Gamma(\gamma)}\left(I^{\alpha} v_{n}(s)(b)-\sum_{i=1}^{m} \delta_{i} I^{\alpha+\varphi_{i}} v_{n}(s)\left(\xi_{i}\right)\right) .
$$

Thus it suffices to show that there exists $v_{*} \in S_{F, x_{*}}$ such that for each $t \in[a, b]$,

$$
h_{*}(t)=I^{\alpha} v(s)(t)+\frac{(t-a)^{\gamma-1}}{\Lambda \Gamma(\gamma)}\left(I^{\alpha} v_{*}(s)(b)-\sum_{i=1}^{m} \delta_{i} I^{\alpha+\varphi_{i}} v_{*}(s)\left(\xi_{i}\right)\right) .
$$

Let us consider the linear operator $\Theta: L^{1}(J, \mathbb{R}) \rightarrow C(J, \mathbb{R})$ given by

$$
v \mapsto \Theta(v)(t)=I^{\alpha} v(s)(t)+\frac{(t-a)^{\gamma-1}}{\Lambda \Gamma(\gamma)}\left(I^{\alpha} v(s)(b)-\sum_{i=1}^{m} \delta_{i} I^{\alpha+\varphi_{i}} v(s)\left(\xi_{i}\right)\right) .
$$

Observe that $\left\|h_{n}(t)-h_{*}(t)\right\| \rightarrow 0$, as $n \rightarrow \infty$. Therefore, it follows by a Lazota-Opial result [23], that $\Theta \circ S_{F}$ is a closed graph operator. Further, we have $h_{n}(t) \in \Theta\left(S_{F, x_{n}}\right)$. Since $x_{n} \rightarrow x_{*}$, we have

$$
h_{*}(t)=I^{\alpha} v_{*}(s)(t)+\frac{(t-a)^{\gamma-1}}{\Lambda \Gamma(\gamma)}\left(I^{\alpha} v_{*}(s)(b)-\sum_{i=1}^{m} \delta_{i} I^{\alpha+\varphi_{i}} v_{*}(s)\left(\xi_{i}\right)\right),
$$

for some $v_{*} \in S_{F, x_{*}}$.

Step 5. We show there exists an open set $U \subseteq C([a, b], \mathbb{R})$ with $x \notin v \mathcal{F}(x)$ for any $v \in(0,1)$ and all $y \in \partial U$.

Let $v \in(0,1)$ and $x \in v \mathcal{F}(x)$. Then there exists $v \in L^{1}([a, b], \mathbb{R})$ with $v \in S_{F, x}$ such that, for $t \in[a, b]$, we have

$$
x(t)=v I^{\alpha} v(s)(t)+v \frac{(t-a)^{\gamma-1}}{\Lambda \Gamma(\gamma)}\left(I^{\alpha} v(s)(b)-\sum_{i=1}^{m} \delta_{i} I^{\alpha+\varphi_{i}} v(s)\left(\xi_{i}\right)\right),
$$


As in the second step, it can be shown that

$$
|x(t)| \leq\|p\| \psi(\|x\|)\left\{\frac{(b-a)^{\alpha}}{\Gamma(\alpha+1)}+\frac{(b-a)^{\gamma-1}}{|\Lambda| \Gamma(\gamma)}\left(\frac{(b-a)^{\alpha}}{\Gamma(\alpha+1)}+\sum_{i=1}^{m}\left|\delta_{i}\right| \frac{\left(\xi_{i}-a\right)^{\alpha+\varphi_{i}}}{\Gamma\left(\alpha+\varphi_{i}+1\right)}\right)\right\} .
$$

which implies that

$$
\|x\| \leq\|p\| \psi(\|x\|)\left\{\frac{(b-a)^{\alpha}}{\Gamma(\alpha+1)}+\frac{(b-a)^{\gamma-1}}{|\Lambda| \Gamma(\gamma)}\left(\frac{(b-a)^{\alpha}}{\Gamma(\alpha+1)}+\sum_{i=1}^{m}\left|\delta_{i}\right| \frac{\left(\xi_{i}-a\right)^{\alpha+\varphi_{i}}}{\Gamma\left(\alpha+\varphi_{i}+1\right)}\right)\right\} .
$$

Consequently

$$
\frac{\|x\|}{\|p\| \psi(\|x\|)\left\{\frac{(b-a)^{\alpha}}{\Gamma(\alpha+1)}+\frac{(b-a)^{\gamma-1}}{|\Lambda| \Gamma(\gamma)}\left(\frac{(b-a)^{\alpha}}{\Gamma(\alpha+1)}+\sum_{i=1}^{m}\left|\delta_{i}\right| \frac{\left(\xi_{i}-a\right)^{\alpha+\varphi_{i}}}{\Gamma\left(\alpha+\varphi_{i}+1\right)}\right)\right\}} \leq 1 .
$$

In view of $\left(H_{3}\right)$, there exists $M$ such that $\|x\| \neq M$. Let us set

$$
U=\{x \in C([a, b], \mathbb{R}):\|x\|<M\} .
$$

Note that the operator $\mathcal{F}: \bar{U} \rightarrow \mathcal{P}(C([a, b], \mathbb{R}))$ is a compact multivalued map, u.s.c. with convex closed values. From the choice of $U$, there is no $x \in \partial U$ such that $x \in v \mathcal{F}(x)$ for some $v \in(0,1)$. In consequence, we deduce by the nonlinear alternative of Leray-Schauder type [22] that $\mathcal{F}$ has a fixed point $y \in \bar{U}$ which is a solution of the problem (3) and (4). This completes the proof.

In our second result, we show the existence of solutions for the problem (3) and (4) when $F$ is not necessary nonconvex valued by applying a fixed point theorem for multivalued maps due to Nadler [24]. Let us first describe the necessary material needed to establish this result.

Let $(X, d)$ be a metric space induced from the normed space $(X ;\|\cdot\|)$. Consider $H_{d}: \mathcal{P}(X) \times$ $\mathcal{P}(X) \rightarrow \mathbb{R} \cup\{\infty\}$ given by

$$
H_{d}(A, B)=\max \left\{\sup _{a \in A} d(a, B), \sup _{b \in B} d(A, b)\right\}
$$

where $d(A, b)=\inf _{a \in A} d(a ; b)$ and $d(a, B)=\inf _{b \in B} d(a ; b)$. Then $\left(\mathcal{P}_{c l, b}(X), H_{d}\right)$ is a metric space (see [25]).

Definition 7. A multivalued operator $N: X \rightarrow \mathcal{P}_{c l}(X)$ is called

(a) $\theta$-Lipschitz if and only if there exists $\theta>0$ such that

$$
H_{d}(N(x), N(y)) \leq \theta d(x, y) \text { for each } x, y \in X ;
$$

(b) a contraction if and only if it is $\theta$-Lipschitz with $\theta<1$.

Lemma 4 ([24]). Let $(X, d)$ be a complete metric space. If $N: X \rightarrow \mathcal{P}_{b, c l}(X)$ is a multivalued contraction then $N$ has a fixed point.

Theorem 2. Assume that the following conditions hold:

$\left(A_{1}\right) F:[a, b] \times \mathbb{R} \rightarrow \mathcal{P}_{c p}(\mathbb{R})$ is such that $F(\cdot, x):[a, b] \rightarrow \mathcal{P}_{c p}(\mathbb{R})$ is measurable for each $x \in \mathbb{R}$.

$\left(A_{2}\right) H_{d}(F(t, x), F(t, \bar{x})) \leq m(t)|x-\bar{x}|$ for almost all $t \in J$ and $x, \bar{x} \in \mathbb{R}$ with $m \in C\left([a, b], \mathbb{R}^{+}\right)$and $d(0, F(t, 0)) \leq m(t)$ for almost all $t \in[a, b]$. 
Then the boundary value problem (3) and (4) has at least one solution on $[a, b]$ if

$$
\delta:=\left\{\frac{(b-a)^{\alpha}}{\Gamma(\alpha+1)}+\frac{(b-a)^{\gamma-1}}{|\Lambda| \Gamma(\gamma)}\left(\frac{(b-a)^{\alpha}}{\Gamma(\alpha+1)}+\sum_{i=1}^{m}\left|\delta_{i}\right| \frac{\left(\xi_{i}-a\right)^{\alpha+\varphi_{i}}}{\Gamma\left(\alpha+\varphi_{i}+1\right)}\right)\right\}\|m\|<1 .
$$

Proof. Consider the operator $\mathcal{F}$ defined by (9). Observe that the set $S_{F, x}$ is nonempty for each $y \in C([a, b], \mathbb{R})$ by the assumption $\left(A_{1}\right)$, so $F$ has a measurable selection (see Theorem III.6 [26]). Now we show that the operator $\mathcal{F}$ satisfies the assumptions of Nadler theorem [24]. We show that $\mathcal{F}(x) \in \mathcal{P}_{c l}(C([a, b], \mathbb{R}))$ for each $x \in C([a, b], \mathbb{R})$. Let $\left\{u_{n}\right\}_{n \geq 0} \in \mathcal{F}(x)$ be such that $u_{n} \rightarrow u(n \rightarrow \infty)$ in $C([a, b], \mathbb{R})$. Then $u \in C([a, b], \mathbb{R})$ and there exists $v_{n} \in S_{F, x_{n}}$ such that, for each $t \in[a, b]$,

$$
u_{n}(t)=I^{\alpha} v_{n}(s)(t)+\frac{(t-a)^{\gamma-1}}{\Lambda \Gamma(\gamma)}\left(I^{\alpha} v_{n}(s)(b)-\sum_{i=1}^{m} \delta_{i} I^{\alpha+\varphi_{i}} v_{n}(s)\left(\xi_{i}\right)\right)
$$

As $F$ has compact values, we pass onto a subsequence (if necessary) to obtain that $v_{n}$ converges to $v$ in $L^{1}([a, b], \mathbb{R})$. Thus, $v \in S_{F, x}$ and for each $t \in J$, we have

$$
u_{n}(t) \rightarrow u(t)=I^{\alpha} v(s)(t)+\frac{(t-a)^{\gamma-1}}{\Lambda \Gamma(\gamma)}\left(I^{\alpha} v(s)(b)-\sum_{i=1}^{m} \delta_{i} I^{\alpha+\varphi_{i}} v(s)\left(\xi_{i}\right)\right) .
$$

Hence, $u \in \mathcal{F}(x)$.

Next we show that there exists $\delta<1$ (defined by (10)), such that

$$
H_{d}(\mathcal{F}(x), \mathcal{F}(\bar{x})) \leq \delta\|x-\bar{x}\| \text { for each } x, \bar{x} \in C^{2}([a, b], \mathbb{R}) .
$$

Let $x, \bar{x} \in C^{2}([a, b], \mathbb{R})$ and $h_{1} \in \mathcal{F}(x)$. Then there exists $v_{1}(t) \in F(t, x(t))$ such that, for each $t \in[a, b]$,

$$
h_{1}(t)=I^{\alpha} v_{1}(s)(t)+\frac{(t-a)^{\gamma-1}}{\Lambda \Gamma(\gamma)}\left(I^{\alpha} v_{1}(s)(b)-\sum_{i=1}^{m} \delta_{i} I^{\alpha+\varphi_{i}} v_{1}(s)\left(\xi_{i}\right)\right) .
$$

By $\left(A_{2}\right)$, we have

$$
H_{d}(F(t, x), F(t, \bar{x})) \leq m(t)|x(t)-\bar{x}(t)| .
$$

Therefore, there exists $w \in F(t, \bar{x}(t))$, such that

$$
\left|v_{1}(t)-w\right| \leq m(t)|x(t)-\bar{x}(t)|, \quad t \in[a, b] .
$$

Define $U: J \rightarrow \mathcal{P}(\mathbb{R})$ by

$$
U(t)=\left\{w \in \mathbb{R}:\left|v_{1}(t)-w\right| \leq m(t)|x(t)-\bar{x}(t)|\right\} .
$$

Since the multivalued operator $U \cap F(t, \bar{x}(t))$ is measurable (Proposition III.4 [26]), there exists a function $v_{2}(t)$ which is a measurable selection for $U$. Therefore, $v_{2}(t) \in F(t, \bar{x}(t))$ and for each $t \in[a, b]$, we have $\left|v_{1}(t)-v_{2}(t)\right| \leq m(t)|x(t)-\bar{x}(t)|$.

For each $t \in[a, b]$, let us define

$$
h_{2}(t)=I^{\alpha} v_{2}(s)(t)+\frac{(t-a)^{\gamma-1}}{\Lambda \Gamma(\gamma)}\left(I^{\alpha} v_{2}(s)(b)-\sum_{i=1}^{m} \delta_{i} I^{\alpha+\varphi_{i}} v_{2}(s)\left(\xi_{i}\right)\right) .
$$

Thus,

$$
\begin{aligned}
& \left|h_{1}(t)-h_{2}(t)\right| \\
\leq & I^{\alpha}\left(\left|v_{1}(s)-v_{2}(s)\right|\right)(t)
\end{aligned}
$$




$$
\begin{aligned}
& +\frac{(b-a)^{\gamma-1}}{\Lambda \Gamma(\gamma)}\left(I^{\alpha}\left(\left|v_{1}(s)-v_{2}(s)\right|\right)(b)+\sum_{i=1}^{m} \delta_{i} I^{\alpha+\varphi_{i}}\left(\left|v_{1}(s)-v_{2}(s)\right|\right)\left(\xi_{i}\right)\right) \\
\leq & \left\{\frac{(b-a)^{\alpha}}{\Gamma(\alpha+1)}+\frac{(b-a)^{\gamma-1}}{|\Lambda| \Gamma(\gamma)}\left(\frac{(b-a)^{\alpha}}{\Gamma(\alpha+1)}+\sum_{i=1}^{m}\left|\delta_{i}\right| \frac{\left(\xi_{i}-a\right)^{\alpha+\varphi_{i}}}{\Gamma\left(\alpha+\varphi_{i}+1\right)}\right)\right\}\|m\|\|x-\bar{x}\| .
\end{aligned}
$$

Hence,

$$
\left\|h_{1}-h_{2}\right\| \leq\left\{\frac{(b-a)^{\alpha}}{\Gamma(\alpha+1)}+\frac{(b-a)^{\gamma-1}}{|\Lambda| \Gamma(\gamma)}\left(\frac{(b-a)^{\alpha}}{\Gamma(\alpha+1)}+\sum_{i=1}^{m}\left|\delta_{i}\right| \frac{\left(\xi_{i}-a\right)^{\alpha+\varphi_{i}}}{\Gamma\left(\alpha+\varphi_{i}+1\right)}\right)\right\}\|m\|\|x-\bar{x}\| .
$$

Analogously, interchanging the roles of $x$ and $\bar{x}$, we obtain

$$
\begin{aligned}
& H_{d}(\mathcal{F}(x), \mathcal{F}(\bar{x})) \\
\leq & \left\{\frac{(b-a)^{\alpha}}{\Gamma(\alpha+1)}+\frac{(b-a)^{\gamma-1}}{|\Lambda| \Gamma(\gamma)}\left(\frac{(b-a)^{\alpha}}{\Gamma(\alpha+1)}+\sum_{i=1}^{m}\left|\delta_{i}\right| \frac{\left(\xi_{i}-a\right)^{\alpha+\varphi_{i}}}{\Gamma\left(\alpha+\varphi_{i}+1\right)}\right)\right\}\|m\|\|x-\bar{x}\| .
\end{aligned}
$$

Therefore, $\mathcal{F}$ is a contraction. Therefore, it follows by Nadler theorem [24] that $\mathcal{F}$ has a fixed point $x$ which is a solution of (3) and (4). This completes the proof.

\section{Examples}

Example 1. Consider the nonlocal boundary value problem with Hilfer fractional differential inclusion of the form:

$$
\left\{\begin{array}{l}
{ }^{H} D^{\frac{3}{2}, \frac{1}{2}} x(t) \in F(t, x(t)), \quad t \in\left[\frac{1}{4}, \frac{3}{2}\right], \\
x\left(\frac{1}{4}\right)=0, \quad x\left(\frac{3}{2}\right)=\frac{2}{3} I^{\frac{1}{2}} x\left(\frac{1}{2}\right)+\frac{3}{4} I^{\frac{3}{2}} x\left(\frac{3}{4}\right)+\frac{4}{5} I^{\frac{5}{2}} x(1)+\frac{5}{6} I^{\frac{7}{2}} x\left(\frac{5}{4}\right) .
\end{array}\right.
$$

Here, $\alpha=3 / 2, \beta=1 / 2, a=1 / 4, b=3 / 2, m=4, \delta_{i}=(i+1) /(i+2), \varphi_{i}=(2 i-1) / 2$ and $\xi_{i}=(i+1) / 4, i=1,2,3,4$. From all information, we can find that $\gamma=7 / 4,|\Lambda| \approx 1.058845070$ and

$$
\frac{(b-a)^{\alpha}}{\Gamma(\alpha+1)}+\frac{(b-a)^{\gamma-1}}{|\Lambda| \Gamma(\gamma)}\left(\frac{(b-a)^{\alpha}}{\Gamma(\alpha+1)}+\sum_{i=1}^{m}\left|\delta_{i}\right| \frac{\left(\xi_{i}-a\right)^{\alpha+\varphi_{i}}}{\Gamma\left(\alpha+\varphi_{i}+1\right)}\right) \approx 1.116843293
$$

(i) Let the set $F(t, x)$ be given by

$$
F(t, x)=\left[\frac{4}{4 t+7}\left(\frac{x^{2}+2|x|}{1+|x|}+\frac{2}{3}\right), \frac{4}{4 t+11}\left(\frac{e^{-|x|} \sin x^{2}}{x^{4}+1}+\frac{1}{4}\right)\right] .
$$

From (12), we have

$$
\|F(t, x)\|_{\mathcal{P}} \leq\left(\frac{4}{4 t+7}\right)\left(\|x\|+\frac{5}{3}\right),
$$

which can be set $p(t)=4 /(4 t+7)$ and $\psi(x)=x+(5 / 3)$. Then we have $\|p\|=1 / 2$ and there exists a constant $M>2.107672935$ satisfying inequality in $\left(H_{3}\right)$. Therefore, by the benefit of Theorem (1), the boundary value problem (11) with (12) has at least one solution on $[1 / 4,3 / 2]$.

(ii) The set $F(t, x)$ is defined by

$$
F(t, x)=\left[\left(\frac{t+1}{4}\right)\left(\tan ^{-1}|x|+\frac{1}{5}\right),\left(\frac{1+\sin t}{6}\right)\left(\frac{x^{2}+|x|}{2(1+|x|)}+\frac{1}{4}\right)\right] .
$$


It is clear that $F$ defined in (13) is measurable for all $x \in \mathbb{R}$. Next, we see that

$$
H_{d}(F(t, x), F(t, y)) \leq \frac{1}{4}(t+1)|x-y|
$$

for all $x, y \in \mathbb{R}$. Setting $m(t)=(t+1) / 4$, we have $\|m\|=5 / 8$ and $d(0, F(t, 0)) \leq m(t)$ for all $t \in[1 / 4,3 / 2]$. Then we have

$$
\left\{\frac{(b-a)^{\alpha}}{\Gamma(\alpha+1)}+\frac{(b-a)^{\gamma-1}}{|\Lambda| \Gamma(\gamma)}\left(\frac{(b-a)^{\alpha}}{\Gamma(\alpha+1)}+\sum_{i=1}^{m}\left|\delta_{i}\right| \frac{\left(\xi_{i}-a\right)^{\alpha+\varphi_{i}}}{\Gamma\left(\alpha+\varphi_{i}+1\right)}\right)\right\}\|m\| \approx 0.6980270581<1
$$

Hence, by applying Theorem 2, we deduce that the boundary value problem (11) with (13) has at least one solution on $[1 / 4,3 / 2]$.

\section{Conclusions}

We initiated in this work the study of boundary value problems for Hilfer fractional differential inclusions. We obtain existence results by considering the cases when the multivalued map has convex or nonconvex values. In the case of convex multivalued map the existence result is proved via nonlinear alternative of Leray-Schauder type, while in the case of nonconvex multivalued map the existence result is proved by using a fixed point theorem due to Nadler and concern multivalued contractions. The obtained results are well illustrated by numerical examples. We emphasize that our results are new in the context of Hilfer fractional differential inclusions and contribute significantly to the existing literature on the topic.

Author Contributions: Conceptualization, S.K.N. and J.T.; methodology, A.W., S.K.N., and J.T.; formal analysis, A.W., S.K.N., and J.T. All authors have read and agreed to the published version of the manuscript.

Funding: This research was funded by King Mongkut's University of Technology North Bangkok. Contract no. KMUTNB-62-KNOW-42.

Acknowledgments: The authors thanks the referees for their helpful suggestions which improved final version of this paper.

Conflicts of Interest: The authors declare no conflict of interest.

\section{References}

1. Diethelm, K. The Analysis of Fractional Differential Equations; Lecture Notes in Mathematics; Springer: New York, NY, USA, 2010.

2. Kilbas, A.A.; Srivastava, H.M.; Trujillo, J.J. Theory and Applications of the Fractional Differential Equations; North-Holland Mathematics Studies: Amsterdam, The Netherlands, 2006.

3. Lakshmikantham, V.; Leela, S.; Devi, J.V. Theory of Fractional Dynamic Systems; Cambridge Scientific Publishers: Cambridge, UK, 2009.

4. Miller, K.S.; Ross, B. An Introduction to the Fractional Calculus and Differential Equations; John Wiley: New York, NY, USA, 1993.

5. Podlubny, I. Fractional Differential Equations; Academic Press: New York, NY, USA, 1999.

6. Samko, S.G.; Kilbas, A.A.; Marichev, O.I. Fractional Integrals and Derivatives; Gordon and Breach Science: Yverdon, Switzerland, 1993.

7. Zhou, Y. Basic Theory of Fractional Differential Equations; World Scientific: Singapore, 2014.

8. Martinez-Garcia, M.; Kalawsky, R.S.; Gordon, T.; Smith, T.; Flemisch, Q.M.F. Communication and Interaction with semiautonomous ground vehicles by force control steering. IEEE Trans. Cybern. 2020. [CrossRef] [PubMed]

9. Martinez-Garcia, M.; Zhang, Y.; Gordon, T. Memory pattern identification for feedback tracking control in human-machine systems. Hum. Factors 2019. [CrossRef] [PubMed]

10. Hilfer, R. (Ed.) Applications of Fractional Calculus in Physics; World Scientific: Singapore, 2000.

11. Hilfer, R. Experimental evidence for fractional time evolution in glass forming materials. J. Chem. Phys. 2002, 284, 399-408. [CrossRef] 
12. Hilfer, R.; Luchko, Y.; Tomovski, Z. Operational method for the solution of fractional differential equations with generalized Riemann-Liouvill fractional derivatives. Frac. Calc. Appl. Anal. 2009, 12, $299-318$.

13. Furati, K.M.; Kassim, N.D.; Tatar, N.E. Existence and uniqueness for a problem involving Hilfer fractional derivative. Comput. Math. Appl. 2012, 64, 1616-1626. [CrossRef]

14. Gu, H.; Trujillo, J.J. Existence of mild solution for evolution equation with Hilfer fractional derivative. Appl. Math. Comput. 2015, 257, 344-354. [CrossRef]

15. Wang, J.; Zhang, Y. Nonlocal initial value problems for differential equations with Hilfer fractional derivative. Appl. Math. Comput. 2015, 266, 850-859. [CrossRef]

16. Asawasamrit, S.; Kijjathanakorn, A.; Ntouyas, S.K.; Tariboon, J. Nonlocal boundary value problems for Hilfer fractional differential equations. Bull. Korean Math. Soc. 2018, 55, 1639-1657.

17. Wongcharoen, A.; Ntouyas, S.K.; Tariboon, J. Nonlocal boundary value problems for Hilfer type pantograph fractional differential equations and inclusions. Adv. Differ. Equ. 2020, 2020, 279. [CrossRef]

18. Wongcharoen, A.; Ntouyas, S.K.; Tariboon, J. On coupled systems for Hilfer fractional differential equations with nonlocal integral boundary conditions. J. Math. 2020, 2020, 2875152. [CrossRef]

19. Deimling, K. Multivalued Differential Equations; Walter De Gruyter: Berlin, Germany; New York, NY, USA, 1992.

20. Hu, S.; Papageorgiou, N. Handbook of Multivalued Analysis, Theory I; Kluwer: Dordrecht, The Netherlands, 1997.

21. Ahmad, B.; Alsaedi, A.; Ntouyas, S.K.; Tariboon, J. Hadamard-Type Fractional Differential Equations, Inclusions and Inequalities; Springer: Cham, Switzerland, 2017.

22. Granas, A.; Dugundji, J. Fixed Point Theory; Springer: New York, NY, USA, 2005.

23. Lasota, A.; Opial, Z. An application of the Kakutani-Ky Fan theorem in the theory of ordinary differential equations. Bull. Acad. Polon. Sci. Ser. Sci. Math. Astronom. Phys. 1965, 13, 781-786.

24. Nadler, S.B., Jr. Multivalued contraction mappings. Pasific J. Math. 1969, 30, 475-488. [CrossRef]

25. Kisielewicz, M. Differential Inclusions and Optimal Control; Kluwer: Dordrecht, The Netherlands, 1991.

26. Castaing, C.; Valadier, M. Convex Analysis and Measurable Multifunctions; Lecture Notes in Mathematics 580; Springer: Berlin/Heidelberg, Germany; New York, NY, USA, 1977.

Publisher's Note: MDPI stays neutral with regard to jurisdictional claims in published maps and institutional affiliations.

(C) 2020 by the authors. Licensee MDPI, Basel, Switzerland. This article is an open access article distributed under the terms and conditions of the Creative Commons Attribution (CC BY) license (http:/ / creativecommons.org/licenses/by/4.0/). 